\title{
A time domain sampling method for inverse acoustic scattering problems
}

\author{
Yukun Guo, Dietmar Hömbergł, Guanghui Huł Jingzhi Li ${ }^{\S}$ and Hongyu Liu
}

March 23, 2016

\begin{abstract}
This work concerns the inverse scattering problems of imaging unknown/inaccessible scatterers by transient acoustic near-field measurements. Based on the analysis of the migration method, we propose efficient and effective sampling schemes for imaging small and extended scatterers from knowledge of time-dependent scattered data due to incident impulsive point sources. Though the inverse scattering problems are known to be nonlinear and ill-posed, the proposed imaging algorithms are totally "direct" involving only integral calculations on the measurement surface. Theoretical justifications are presented and numerical experiments are conducted to demonstrate the effectiveness and robustness of our methods. In particular, the proposed static imaging functionals enhance the performance of the total focusing method (TFM) and the dynamic imaging functionals show analogous behavior to the time reversal inversion but without solving time-dependent wave equations.
\end{abstract}

Key words. inverse acoustic scattering, sampling method, time domain, dynamic measurements, Kirchhoff migration

\section{Introduction}

The inverse scattering theory of acoustic waves and the corresponding reconstruction methods have been extensively studied in recent years. Considerable developments have been achieved to the inverse scattering problem of determining the location and shape of a scattering object from the knowledge of acoustic scattered waves corresponding to time-harmonic incident waves. Those methods are usually referred to as the frequency-domain methods in the literature; see, e.g., $[3,10,20]$ and the references therein.

Among various frequency-domain methods for inverse scattering problems, we are particularly interested in the so-called sampling-type methods. The core

\footnotetext{
* Department of Mathematics, Harbin Institute of Technology, Harbin, P. R. China. Email: ykguo@hit.edu.cn

${ }^{\dagger}$ Weierstrass Institute, Berlin, Germany. Email: Dietmar.Hoemberg@wias-berlin.de

${ }^{\ddagger}$ Weierstrass Institute, Berlin, Germany. Email: hu@wias-berlin.de

$\S$ Department of Mathematics, Southern University of Science and Technology, Shenzhen, P. R. China. Email: li.jz@sustc.edu.cn. Corresponding author

『Department of Mathematics, Hong Kong Baptist University, Kowloon, Hong Kong SAR. Email: hongyuliu@hkbu.edu.hk
} 
of a sampling-type method is a certain imaging/indicator functional, which is obtained by using the measurement data and can be used to indicate whether a point (or a line, or a surface) in the space belongs to the interior or exterior of the scatterer. In general, the imaging functionals are easily computed without iterations required, though the inverse scattering problems are known to be nonlinear and ill-conditioned. Moreover, the sampling-type methods usually require very little a priori information of the unknown/inaccessible target scatterer. Due to its practical importance, the sampling-type methods has drawn a great deal of interest in the literature, and examples include the linear sampling method [9], the factorization method [20], the point source and probe method [27], the enclosure method [16], the MUSCI-type method [2, 3] and the recent one-shot and orthogonality sampling methods [23, 24, 28], among others. Generally, the frequency-domain sampling methods could work with the data corresponding to only a single frequency.

However, in diverse practical applications such as sonar detection, geophysical exploration, medical imaging and nondestructive testing, dynamic measurement data are usually easy to obtain. Therefore, imaging unknown/inaccessible scatterers through the time-dependent acoustic waves is a topic of significant interest to these areas. There exist a number of reconstruction algorithms in the time domain such as the time reversal techniques $[11,13,14]$, the reversed time migration [4], the boundary control method [5, 26].In a series of recent publications, such as $[6,7,29,19]$ and references cited there, two globally convergent numerical methods for inverse scattering problem in the time domain were proposed. In this approach the spatially distributed speed $c(x), x \in \mathbb{R}^{3}$ of the acoustic signal is unknown. Furthermore, the global convergence theory was firmly confirmed on experimental data, see, e.g. [7, 29] and references cited there. In particular the so-called Stolt migration method was successfully applied in [29] to propagate the experimentally measured data closer to the unknown targets". For sampling type methods using dynamic measurements, we refer to the point source method [25], the probe method [8], the linear sampling method [12], the enclosure method [17], the multiple signal classification (MUSIC) method [21] and the total focusing method (TFM) arising from nondestructive evaluation [15]. Note that the TFM is sometimes described as the "gold standard" in the classical beamforming, and it shares the same idea of the so-called Kirchhoff migration widely used in geophysics. Both of them form the image with the superposition of the scattered signals incited by each transducer. They are robust to measurement noise but sensitive to medium noise [1] . In a series of works $[1,14]$, correlation-based imaging schemes have been justified to recover the position of a point reflector in a noisy environment.

The goal of this paper is to show that the TFM and Kirchhoff migration can be used for imaging not only acoustic scatterers with small inclusions but also the shape of regular-size/extended penetrable and impenetrable scatterers with multiple components, including cracks. The proposed method can be regarded as a strengthened version of the total focusing method or Kirchhoff migration in a stationary medium. To our best knowledge, they have been so far applied to the reconstructions of point-like scatterers only. Using synthetic data, in this work we apply for the first time the newly developed strengthened versions of TFM to imaging crack-like and regular-size/extended scatterers with multiple components. In particular, the newly proposed dynamic imaging/indicator functionals show analogous behavior of the time reversal inversion but with- 
out having to solve time-dependent wave equations. The promising and salient features of our time-domain sampling method can be summarized as follows. First, the imaging/indicator functionals are formulated directly from the time domain data. No Fourier or Laplace transform is needed on the algorithm level. Second, the imaging schemes do not require the strong a priori information of the physical properties of the scatterer, and apply to both penetrable medium and impenetrable obstacle. There is no need to know the type of boundary conditions of an impenetrable obstacle. Third, the imaging schemes are explicit because the imaging indicators do not rely on any matrix inversion or forward solution process. Hence, the method is very robust with respect to measurement noise. Finally, the method is very easy to implement with computational efficiency. Only cheap integrations are involved in calculating the imaging/indicator functionals. In this work, we shall present some mathematical analysis in justifying the indicator behavior of the proposed imaging functionals in the scenario with point-like scatterers. Extensive numerical experiments in two and three dimensions are conducted to demonstrate the feasibility and robustness of our methods. Using the correlation-based algorithms [1, 14], we believe that our approach also applies to extended scatterers in a random medium.

The rest of the paper is organized as follows. In the next section we give a brief description of the forward and inverse scattering problems that we are concerned with. Single-source and multi-source indicator/imaging functionals are introduced in Section 3. The behavior of the imaging indicators for pointlike scatterers will also be analyzed in this section. Section 4 is devoted to numerical experiments and the paper is concluded in Section 5.

\section{Problem setting}

Let $D \subset \mathbb{R}^{N}(N=2,3)$ be an open bounded domain with the Lipschitz boundary $\partial D$ and connected exterior $\mathbb{R}^{N} \backslash \bar{D}$. D represents a stationary scatterer for our study. It is assumed that the exterior $\mathbb{R}^{N} \backslash \bar{D}$ is an isotropic and homogeneous background medium with a constant phase velocity $c_{0} \in \mathbb{R}_{+}$. Let $\Gamma_{i} \subset \mathbb{R}^{N} \backslash \bar{D}$ be a closed Lipschitz surface on which point sources emit incident waves for the probing of the scatterer. Throughout, we assume that the incident wave $u_{\chi}^{i}(x, t ; y)$ with $x, y \in \mathbb{R}^{N}, t>0$ is a monopole emitted at the source location $y \in \Gamma_{i}$ with a temporal pulse signal $\chi: \mathbb{R} \rightarrow \mathbb{R}$ such that $\chi(t) \equiv 0$ for $t<0$. That is, $u_{\chi}^{i}(\cdot, \cdot ; y)$ is a causal solution to the following wave propagation problem in the free space

$$
\begin{aligned}
\Delta u(x, t)-c_{0}^{-2} \partial_{t t} u(x, t) & =-\delta(x-y) \partial_{t} \chi(t) & & \text { in } \mathbb{R}^{N} \times \mathbb{R}^{+}, \\
u(\cdot, 0) & =\partial_{t} u(\cdot, 0)=0 & & \text { in } \mathbb{R}^{N},
\end{aligned}
$$

where $\delta$ is the Dirac delta distribution. In particular, if $\chi(t)$ is chosen as the Heaviside function $H(t)$, then it is well known that the unique solution to problem (1)-(2) is given by

$$
\Phi(x, t ; y):= \begin{cases}\frac{H\left(t-c_{0}^{-1}|x-y|\right)}{2 \pi \sqrt{t^{2}-c_{0}^{-2}|x-y|^{2}}}, & N=2, \\ \frac{\delta\left(t-c_{0}^{-1}|x-y|\right)}{4 \pi|x-y|}, & N=3,\end{cases}
$$


which is called the fundamental solution to the wave equation.

Since the excitation signals of Gaussian type are commonly used in a number of practical applications, in this paper we assume that $\chi(t)$ is a causal Gaussianmodulated sinusoidal pulse of the form

$$
\chi(t):= \begin{cases}\sin (\omega t) \exp \left(-\sigma\left(t-\tau_{0}\right)^{2}\right), & t \geq 0, \\ 0, & t<0,\end{cases}
$$

where $\omega>0$ is the center frequency, $\sigma>0$ is the frequency bandwidth parameter, and $\tau_{0}>0$ is the time-shift parameter concerning the pulse peak time. In terms of the center frequency $\omega$, the center wavelength of $\chi(t)$ is defined by $2 \pi c_{0} / \omega$.

Given an incident point source $u^{i}(\cdot, \cdot ; y), y \in \Gamma_{i}$, the direct/forward obstacle scattering problem is to find the scattered field $u^{s}(\cdot, \cdot ; y)$ satisfying the wave equation

$$
\Delta u^{s}-c_{0}^{-2} \partial_{t t} u^{s}=0 \quad \text { in }\left(\mathbb{R}^{N} \backslash \bar{D}\right) \times \mathbb{R}^{+},
$$

the initial conditions

$$
u^{s}(\cdot, 0)=\partial_{t} u^{s}(\cdot, 0)=0 \quad \text { in } \mathbb{R}^{N},
$$

and the boundary condition

$$
\mathfrak{B}\left(u^{i}+u^{s}\right)=0 \quad \text { on } \partial D \times \mathbb{R}^{+} .
$$

The impenetrable obstacle $D$ is referred to as sound-soft (resp. sound-hard) if the pressure (resp. the normal velocity) of the total wave $u=u^{i}+u^{s}$ vanishes on the boundary $\partial D$. Another practical scenario is the one where the pressure and the normal velocity of the total wave is proportional on the boundary, which leads to the impedance boundary condition. Thus the differential operator $\mathfrak{B}$, depending on the physical property of an impenetrable scatterer, takes the form

$$
\mathfrak{B} u:= \begin{cases}\left.u\right|_{\partial D \times \mathbb{R}^{+}}, & \text {if } D \text { is sound-soft, } \\ \left.\partial_{\nu} u\right|_{\partial D \times \mathbb{R}^{+}}, & \text {if } D \text { is sound-hard, } \\ \left.\left(\partial_{\nu} u-\lambda c_{0}^{-1} \partial_{t} u\right)\right|_{\partial D \times \mathbb{R}^{+}}, & \text {if } D \text { is of impedance-type }\end{cases}
$$

where $\nu$ is the unit outward normal vector to $\partial D$ and $\lambda \in C(\partial D)$ is a nonnegative surface impedance function.

If the scatterer is penetrable, then the direct scattering problem can be modelled by the following medium scattering problem: find the scattered field $u^{s}(\cdot, \cdot ; y)$ satisfying the wave equation

$$
c_{0}^{2} \Delta u^{s}-n(x) \partial_{t t} u^{s}=-(1-n(x)) \partial_{t t} u^{i} \quad \text { in } \mathbb{R}^{N} \times \mathbb{R}^{+},
$$

and the initial conditions (5). In (6), the space-variable function $n(x):=$ $c_{0}^{2} / c^{2}(x)$ is the so called refractive index, where the sound speed $c(x)>0$ is a piecewise continuous function such that $c(x)-c_{0}$ has compact support. Then the geometry of the scatterer $D$ is characterized by the compact support of $n(x)-1$. Without loss of generality, we assume that $c_{0} \equiv 1$ in the rest of this paper.

In the present study, the scatterer $D$ does not necessarily occupy a simply connected domain but can be practically very general where $D$ may consist 
of a finite number of disconnected components such that different components possess different shapes, sizes and/or physical properties (penetrable or impenetrable). In what follows, the relative size of each scatterer component is interpreted in terms of the center frequency of the incident pulse. Let $L \in \mathbb{N}$, and let $\Omega_{j}, 1 \leq j \leq L$, be bounded simply connected $C^{2}$ domains in $\mathbb{R}^{N}$ containing the origin. We assume that each reference domain $\Omega_{j}$ is of the unit size, i.e.,

$$
\operatorname{diam}\left(\Omega_{j}\right) \approx 1, \quad 1 \leq j \leq L .
$$

For $\rho_{j} \in \mathbb{R}^{+}, 1 \leq j \leq L$, we define $\rho_{j} \Omega_{j}:=\left\{\rho_{j} x \mid x \in \Omega_{j}\right\}$ and set

$$
D_{j}=z_{j}+\rho_{j} \Omega_{j}, \quad z_{j} \in \mathbb{R}^{N}, 1 \leq j \leq L,
$$

where $z_{j}$ is the location of the $j$-th component $D_{j}$ for $1 \leq j \leq L$. Thus the scatterer $D$ is defined by the union of these components

$$
D:=\bigcup_{j=1}^{L} D_{j} .
$$

A component $D_{j}$ is referred to as a small or point-like scatterer component if its relative/characteristic size is much smaller than the center wavelength of the incident pulse, i.e.,

$$
\omega \rho_{j} \ll 2 \pi \text {. }
$$

A component $D_{j}$ is referred to as an extended scatterer component if its relative/characteristic size is comparable to the center wavelength of the incident pulse, i.e.,

$$
\omega \rho_{j} \sim 2 \pi
$$

Note that an extended scatterer component may be "partially small", for example, $D_{j}$ is a rectangular box which has a small width in proportion to the length or height. If the corresponding reference shape $\Omega_{j}$ of an extended scatterer component $D_{j}$ has a large shape ratio (in terms of the width/length/height), then $D_{j}$ is referred to as a segment-like or crack-like component, otherwise it is referred to as a regular-size component.

If the scatterer consists of multiple point-like and/or extended components, then these components are assumed to be sparsely distributed, i.e.,

$$
\omega \operatorname{dist}\left(z_{j}, z_{j^{\prime}}\right) \gg 2 \pi, \quad \forall j \neq j^{\prime}, 1 \leq j, j^{\prime} \leq L .
$$

The assumption (9) implies that the distance between any two obstacles is much larger than the incident wavelength. Hence, the multiple scattering effects between different scatterer components can be neglected in finite time. Nevertheless, we would like to emphasize that the sparsity assumption would be only needed for the purpose of theoretical analysis. Our numerical examples in Section 4 show that the proposed imaging schemes work for the more general case with different scatterer components being relatively closer to each other. In particular, as long as the distance between different components is bigger than one center wavelength, the proposed imaging schemes would yield satisfactory reconstructions.

Let $\Gamma$ be the closed measurement surface (possible equal to $\Gamma_{i}$ ) such that the scatterer is located inside the domain enclosed by $\Gamma$. Let $T_{0}$ and $T$ denote the 
lower and upper bound of the finite time window for collecting data, respectively. The forward scattering problem can be stated as follows: Given the incident wave $u^{i}(\cdot, \cdot ; y), y \in \Gamma_{i}$ and the scatterer $D$, find the corresponding scattered wave $u^{s}(x, t ; y)$ for $x \in \Gamma$ and $T_{0} \leq t \leq T$. For the well-posedness of the forward problem and the numerical methods for solving time-dependent scattering problems, we refer to the classical book [22, Chapter 4] and the lecture notes by Wilcox [30].

In this paper we will study the inverse problem of recovering the locations of the point-like scatterers and the shape of the extended scatterers from knowledge of the incident wave $u^{i}(\cdot, \cdot ; y)$ and the time-dependent scattered data

$$
\left\{u^{s}(x, t ; y) \mid x \in \Gamma, \quad y \in \Gamma_{i}, \quad T_{0} \leq t \leq T\right\} .
$$

In general, the starting time $T_{0} \geq 0$ can be chosen as the time of the first arrival of the scattered field on $\Gamma$ and the terminal time $T$ can be chosen such that the scattered energy inside $\Gamma$ can be considered as negligible when $t>T$. For ease of exposition, we shall assume below that $T_{0}=0$. We refer to [18] for an overview of the uniqueness and stability results in inverse penetrable and impenetrable obstacle scattering. In particular, a lower bound of the terminal time $T$ was estimated in [18, Theorem 5.1] for ensuring uniqueness in determining a soundsoft or sound-hard stationary obstacle with a single boundary measurement data.

\section{Indicator and imaging functions}

In what follows, given the scattered data, the corresponding time-reversed data is defined by

$$
u^{\operatorname{tr}}(x, t ; y):= \begin{cases}u^{s}(x, T-t ; y), & 0 \leq t \leq T \\ 0, & t<0\end{cases}
$$

for $x \in \Gamma$ and $y \in \Gamma_{i}$. Denote by $G \subset \mathbb{R}^{N}$ a bounded probe region containing the unknown scatterer $D$. For example, one may assume that $G$ is a sub-domain of the region enclosed by $\Gamma$.

\subsection{Single-source indicators}

Let the source position be located at $y \in \Gamma_{i}$. In the present study, we propose the static indicator function with a single point source wave as follows:

$$
I(z ; y):=\int_{0}^{T}\left(\int_{\Gamma} u^{\operatorname{tr}}(x, \tau-|z-x| ; y) \mathrm{d} s(x)\right)^{2} \mathrm{~d} \tau,
$$

where $z \in G$ denotes the sampling point. A dynamic indicator function $\tilde{I}(z, t ; y)$ is defined as

$$
\tilde{I}(z, t ; y):=\int_{0}^{t}\left(\int_{\Gamma} u^{\operatorname{tr}}(x, \tau-|z-x| ; y) \mathrm{d} s(x)\right)^{2} \mathrm{~d} \tau
$$

for $z \in G$ and $t \in(0, T)$. Obviously, $\left.\tilde{I}(z, t ; y)\right|_{t=T}$ coincides with the stationary indicator $I(z ; y)$ defined in (11). The above single-source indicators can be 
used to image multiple point-like scatterers and cracks by plotting the function $z \rightarrow I(z ; y)$ or $z \rightarrow \tilde{I}(z, t ; y)$. It is worthy mentioning that these indicators are in spirit similar to the Kirchhoff migration widely used in geophysics and the total focusing imaging scheme arising from non-destructive testing (see e.g.,[15]) but modified to improve the resolution of the original TFM. Our inspirations are taken from the recent direct sampling methods [28, 24] in the frequency domain. The indicator function of TFM (or Kirchhoff migration) with a single point source takes the form (see e.g. [15]):

$$
I_{\mathrm{TFM}}(z ; y):=\left|\int_{\Gamma} u^{s}\left(x, t_{0}+|y-z|+|x-z| ; y\right) \mathrm{d} s(x)\right|
$$

where $t_{0} \geq 0$ is the peak time of the incident pulse (see (3)). The indicators (11) and (12) differ from (13) in the extra integrations with respect to the parameter $\tau$ over $(0, T)$ or $(0, t)$ and in the square of the integrand. Compared to the above mentioned direct sampling methods in the frequency domain, our indicators involve no inner product and the sampling point is coded in the time variable of the scattered signal. The behavior of the indicator functions (11) and (13) for point-like scatterers is given in the following theorem.

Theorem 1. Let $D \subset \mathbb{R}^{N}$ be a union of point-like scatterers defined by (7) and (8), and let $z_{j}, j=1, \cdots, L$, be the exact locations of the scatterer components. Then there exists an open neighborhood of $z_{j}$, neigh $\left(z_{j}\right), j=1, \cdots, L$, such that $z_{j}$ is a local maximizer of $I(z ; y)$ and $I_{T F M}(z ; y)$ in neigh $\left(z_{j}\right)$. The point $z_{j}$ is also the local maximizer of the dynamic indicator $I(z, t ; y)$ provided $t>$ $T-t_{0}-\left|z_{j}-y\right|$.

Proof. The proof depends on the travel time of the acoustic waves inside the probe region. We first suppose $L=1$, i.e., $D$ consists of a single point-like scatterer located at $z_{1} \in \mathbb{R}^{3}$ only. This suggests that the scattered signal can be regarded as an impulsive source wave emitted from $z_{1}$. Recall that the sound speed has been assumed to be $c_{0}=1$ in the background medium. The wave crest of the incident pulse generated by (3) arrives at the surface $\left\{x \in \mathbb{R}^{N} \mid\right.$ $|x-y|=a\}$ when $t=a+t_{0}$ and will touch the scatterer at $t=\left|z_{1}-y\right|+t_{0}$. Hence, the wave crest of the scattered signal arrives at $\left\{x \in \mathbb{R}^{N}|| x-z_{1} \mid=b\right\}$ when $t=t_{0}+\left|z_{1}-y\right|+b$ for some $b>0$, and will touch the measurement point $x \in \Gamma$ when $t=t_{0}+\left|y-z_{1}\right|+\left|x-z_{1}\right|$. Consequently, we obtain for $z \in \operatorname{neigh}\left(z_{1}\right)$ that

$$
u^{s}\left(x, t_{0}+\left|y-z_{1}\right|+\left|x-z_{1}\right| ; y\right) \geq u^{s}\left(x, t_{0}+|y-z|+|x-z| ; y\right)
$$

uniformly in all $x \in \Gamma$. This proves the relation $I_{\mathrm{TFM}}\left(z_{1} ; y\right) \geq I_{\mathrm{TFM}}(z ; y)$ for all $z \in$ neigh $\left(z_{1}\right)$ when $j=1$. If $D$ consists of several components, the multiple scattering effect between the different point-like scatterers can be neglected due to the assumption (9). Then, by arguing in the same manner one can prove that $z_{j}$ is the local maximizer of $I_{\mathrm{TFM}}(z ; y)$ in neigh $\left(z_{j}\right)$.

Due to the Gaussian pulse excitation (3) and the sparsity assumption (9), the scattered field corresponding to $D$ in a finite time can be regarded as the sum of the scattered data $u_{j}(j=1,2, \cdots, L)$ emitted from the $j$-th scatterer component. On the other hand, there exists $\epsilon>0$ such that $u_{j}(x, t ; y)=0$ if $\left|t-\left(t_{0}+\left|y-z_{j}\right|+\left|x-z_{j}\right|\right)\right|>\epsilon$ since the incident field has a limited bandwidth. 


\begin{tabular}{ll}
\hline Algorithm Locating a multiple point-like scatterers \\
\hline Step 1 1 For a collection of unknown point-like scatterers $D$, select an \\
appropriate source position $y$ and start to send the incident \\
pulse of the form $(3)$ at an initial time $t=0$. \\
Collect the scattered data $u^{s}(\cdot, \cdot ; y)$ at the measurement points \\
and recording time steps between $t=0$ and the (sufficiently \\
long) terminal time $t=T$. \\
Step $3 \quad \begin{array}{l}\text { Select a grid of sampling points in a region containing } D . \text { For } \\
\text { each sampling point } z \text {, calculate the static indicator } I(z ; y) \text { de- } \\
\text { fined in }(11) \text { (alternatively, in addition for each recording time } \\
t, \text { calculate the dynamic indicator } \tilde{I}(z, t ; y) \text { defined in }(12)) \text { ). }\end{array}$ \\
Step 4 $\begin{array}{l}\text { Locate all the significant local maximizers of } I(z ; y) \text { at the sam- } \\
\text { pling grid (alternatively, find the stages of } t \text { when the significant } \\
\text { local maximizers of } \tilde{I}(z, t ; y) \text { at the sampling grid appear, then } \\
\text { locate all the local maximizers at these stages), which represent } \\
\text { locations of the scatterer components of } D .\end{array}$ \\
\hline
\end{tabular}

Therefore, the indicator function $I(z ; y)$ can be reformulated as

$$
I(z ; y)=\sum_{j=1}^{L} I_{j}(z ; y), I_{j}(z ; y):=\int_{t_{j}-\epsilon}^{t_{j}+\epsilon}\left(\int_{\Gamma} u_{j}^{\mathrm{tr}}(x, \tau-|z-x| ; y) \mathrm{d} s(x)\right)^{2} \mathrm{~d} \tau
$$

for some $\epsilon>0$ sufficiently small, with $t_{j}:=T-\left(t_{0}+\left|z_{j}-y\right|\right)$ for $j=1, \cdots, L$. Here $u_{j}^{\text {tr }}$ denotes the time-reversed scattered data emitted from the $j$-th scatterer component, which is defined analogously to (10). Observing that

$$
I_{j}\left(z_{j} ; y\right) \geq I_{j}(z ; y) \quad \text { for } z \in \operatorname{neigh}\left(z_{j}\right) ; \quad I_{j}\left(z_{m} ; y\right)=0 \quad \text { for } m \neq j,
$$

we conclude that

$$
I\left(z_{j} ; y\right) \geq I(z ; y) \quad \text { for all } z \in \operatorname{neigh}\left(z_{j}\right) .
$$

Finally, the behavior of the dynamic indicator (12) follows from (14) and the expression

$$
\tilde{I}(z, t ; y)=\sum_{0<t_{j}<t} I_{j}(z ; y) .
$$

The proof is complete.

Based on Theorem 1, we can readily formulate the scheme for imaging a collection of point-like scatterers.

We end up this section with the following remark.

Remark 1. Compared to the TFM, the integral with respect to $\tau$ in (11) or (12) is necessary because of the Gaussian pulse excitation (3) and the possible existence of multiple scatterer components. Combining this with the square of the integrand in (11) and (12) may improve the imaging resolution of the original TFM. The dynamic indicator shows analogous behavior of the time reversal inversion but without solving time-dependent wave equations. Moreover, it could be used to locate point-like scatterers with weak scattering strength; see Section 4.1 for numerical examples. 


\subsection{Multi-source indicators}

In this section we consider the superposition of single-source indicators for imaging the location and shape of general extended scatterers by sending multiple impulsive point sources. Since the values of (11) at a fixed sampling point $z$ may vary significantly from source to source, a normalization should be taken into account in order to rescale their magnitudes into the same order and thus balance their contributions to the superposition. Recall that $\Gamma_{i}$ denotes the position of the incident point sources. We define the normalized static and dynamic indicators as (cf. (11) or (12))

$$
I(z):=\int_{\Gamma_{i}} \frac{I^{2}(z ; y)}{\max _{z \in G} I^{2}(z ; y)} \mathrm{d} s(y), \quad z \in G,
$$

and

$$
\tilde{I}(z, t):=\int_{\Gamma_{i}} \frac{\tilde{I}^{2}(z, t ; y)}{\max _{z \in G} \tilde{I}^{2}(z, t ; y)} \mathrm{d} s(y), \quad z \in G, \quad 0<t \leq T,
$$

respectively, where $I(z ; y)$ and $\tilde{I}(z, t ; y)$ are the single-source indicators defined in Section 3.1. Meanwhile, we propose another dynamic indicator as following

$$
\tilde{I}_{\mathrm{NEW}}(z, t):=\frac{\tilde{\Lambda}^{2}(z, t)}{\max _{z \in G} \tilde{\Lambda}^{2}(z, t)}, \quad z \in G, \quad 0<t \leq T
$$

with

$$
\tilde{\Lambda}(z, t):=\int_{0}^{t} \int_{\Gamma_{i}}\left(\int_{\Gamma} u^{\operatorname{tr}}(x, \tau-|z-x| ; y) \mathrm{d} s(x)\right)^{2} \mathrm{~d} s(y) \mathrm{d} \tau .
$$

Note that this new indicator $\tilde{I}_{\mathrm{NEW}}(z, t)$ differs from $\tilde{I}(z, t)$ in the orders of normalization and the integration over $\Gamma_{i}$. Our numerics show that $\tilde{I}_{\mathrm{NEW}}(z, t)$ yields more geometrical information of the unknown scatterer than $\tilde{I}(z, t)$. To compare with the TFM, we still need the indicator of the multi-source TFM, given by

$$
I_{\mathrm{TFM}}(z)=\int_{\Gamma_{i}} I_{\mathrm{TFM}}(z ; y) \mathrm{d} s(y), \quad z \in G .
$$

Motivated by the imaging scheme for point-like scatterers, we can analogously formulate the scheme for imaging extended scatterers. Moreover, based on the proof of the Theorem 1 , it is quite reasonable to expect that the values of above multi-source indicators are relatively large when the sampling point $z$ tends to the boundary of the scatterer, namely, the multi-source indicators are capable of imaging general extended scatterers. This will be confirmed in our numerical tests in Section 4.3 and 4.4. However, a theoretical justification of the behavior of these indicators is fraught with difficulties and we shall further explore it in our future work.

\section{$4 \quad$ Numerical examples}

In this section we will give several two and three dimensional numerical examples to demonstrate the feasibility and the effectiveness of the proposed methods. In 


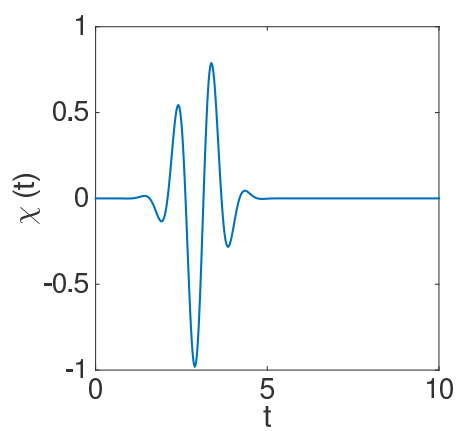

(a)

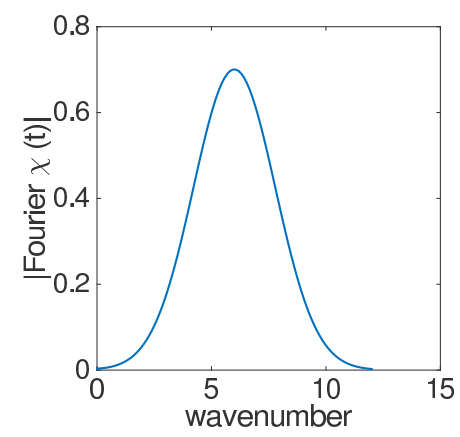

(b)

Figure 1: The incident pulse function. (a) temporal pulse. (b) Fourier spectrum.

the following examples, the pulse

$$
\chi(t):=\sin (6 t) \exp \left(-1.6(t-3)^{2}\right)
$$

was injected at each source point as excitation. The incident pulse function $\chi(t)$ and its wavenumber spectrum (via Fourier transform) are depicted in Figure 1. The center frequency and the center wavelength of $\chi(t)$ are 6 and $\pi / 3$, respectively.

The scattered data were generated using the finite element method and the unbounded exterior domain is truncated by an absorbing boundary condition. The mesh of the forward solver is successively refined until the relatively error of the successive measured scattered data is below $0.1 \%$. To test the stability of the proposed reconstruction scheme, artificial random noise was also added to the synthetic data. The noisy data is given according to the following formula:

$$
u_{\epsilon}^{s}:=u^{s}(1+\epsilon r)
$$

where $\epsilon$ is the noise level and $r$ are uniform random numbers ranging from -1 to 1. In all the examples in the sequel, the scattered data computed by the finite element method was contaminated using $\epsilon=0.1$ before the inversions, namely, the measured data with $10 \%$ noise was used.

Now we specify the discretization in details. Suppose that we have $N_{i} \in \mathbb{N}$ distinct source points $y_{j} \in \Gamma_{i}, j=1, \cdots, N_{i}$. Let's specify the discretization details of the indicator (11) for a source point $y_{j}$. Suppose that we have $N_{m}$ equidistantly distributed receivers $x_{m} \in \Gamma, m=1, \cdots, N_{m}$ and $N_{t}$ equidistant recording time steps $t_{n} \in[0, T], n=1, \cdots, N_{t}$. Then the scattered data is a $N_{m} \times N_{t}$ array $U^{s}$ whose $(m, n)$-th entry is $u^{s}\left(x_{m}, t_{n} ; y_{j}\right)$. The time-reversed data array $U^{t r}$ is obtained by rearranging $U^{s}$ by the order of elements flipped left to right along the rows, namely, the $(m, n)$ entry of $U^{t r}$ is $u^{s}\left(x_{m}, t_{N_{t}-n+1} ; y_{j}\right)$. Afterwards an interpolation is performed at each row of $U^{t r}$ to find the values of time-reversed data at the query time steps, i.e., the data $u^{t r}\left(x_{m}, t_{n}-\mid z-\right.$ $\left.x_{m} \mid ; y_{j}\right), m=1, \cdots, N_{m}, n=1, \cdots, N_{t}$. The integrals in the indicator function are approximated using the trapezoidal rule. Hence, the discretized version of 
indicator (11) can be written as follows

$$
\mathbf{I}\left(z ; y_{j}\right):=\frac{T}{N_{t}-1} \sum_{n=1}^{N_{t}}\left(h_{x} \sum_{m=1}^{N_{m}} u^{\operatorname{tr}}\left(x_{m}, t_{n}-\left|z-x_{m}\right| ; y_{j}\right)\right)^{2}, \quad j=1, \cdots, N_{i}
$$

where $h_{x}$ denotes the distance between two adjacent receivers. Obviously, once the discretized indicator for each single point source is available, the indicator for multiple sources defined in (15) is obtained by direct summation of the normalized single-source indicators, i.e.,

$$
\mathbf{I}(z):=h_{y} \sum_{j=1}^{N_{i}} \frac{\mathbf{I}^{2}\left(z ; y_{j}\right)}{\max _{z \in G} \mathbf{I}^{2}\left(z ; y_{j}\right)}, \quad z \in G
$$

where $h_{y}$ denotes the distance between two adjacent point sources.

The discretized indicator function of the total focusing method can be obtained in the same manner, i.e. (cf. (19) )

$$
\mathbf{I}_{\mathrm{TFM}}(z):=h_{x} h_{y}\left|\sum_{j=1}^{N_{i}} \sum_{m=1}^{N_{m}} u^{s}\left(x_{m}, t_{0}+\left|y_{j}-z\right|+\left|x_{m}-z\right| ; y_{j}\right)\right|, \quad z \in G,
$$

where $t_{0}=\operatorname{argmax}_{t>0}|\chi(t)| \approx 2.88$ is used in the following examples. The dynamic indicator functions (16) and (17) can also be discretized similarly, so we omit the detailed expressions. Finally, we use a uniformly distributed $100 \times 100$ sampling grid over $G:=[-2.5,2.5]^{2}$, or $60 \times 60 \times 60$ sampling grid over $G:=[-3,3]^{3}$ to plot the discretized and normalized indicator function as our reconstructed images. One can also choose a smaller probe region than $G$ depending on the the first arrival time of each receiver.

In the following numerical examples, if not otherwise specified, we use stationary indicator functions that are scaled to the range $[0,1]$ for the sake of comparison.

\subsection{Reconstruction of point-like scatterers with a single source}

In the first example, we aim to reconstruct a collection of point-like scatterers using a single point source. The single source point is chosen as $(-3,0)$. Synthetic scattered data were collected with terminal time $T=16$ and 800 recording time steps. The 48 equidistantly distributed measurement points were placed on the boundary of a square with side length 6 around the sampling region. The true scatterer consists of a sound-soft square with side length 0.1 located at $(-1,2)$, a sound-soft disk with radius 0.05 located at $(0.5,-1.5)$, a sound-hard disk with radius 0.2 located at $(1.5,0)$ and a penetrable medium square with side length 0.3 located at $(-2,-1)$. The sound speed inside the medium square is 5 .

Figure 2 gives the settings of the forward scattering problems and the reconstructions using full and limited aperture observations. We compare the numerical performance of the proposed indicator (11) and the total focusing method (13). As can be seen from Figure 2 (b) (c), with full aperture data acquisition, a local maximum can be clearly visualized at the location of each 


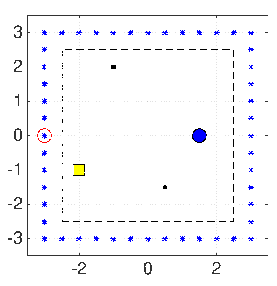

(a)

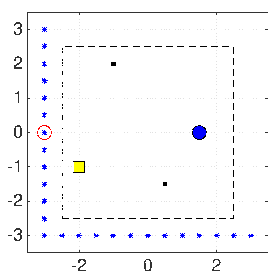

(d)

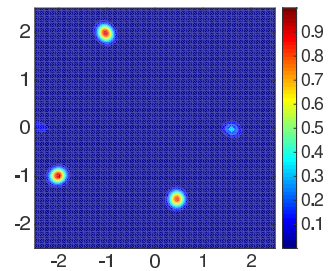

(b)

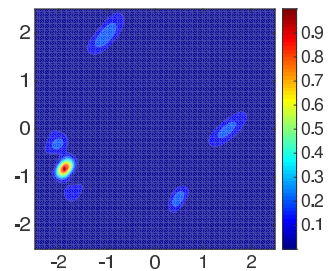

(e)

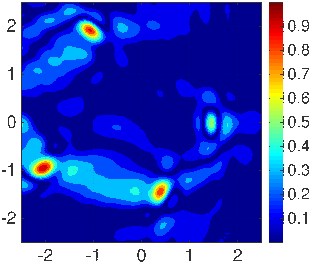

(c)

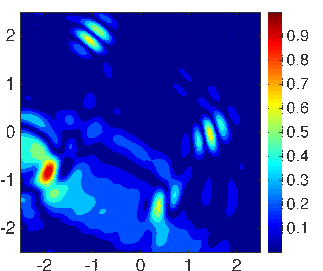

(f)

Figure 2: Reconstruction of small scatterers. Top row: full aperture case. Bottom row: limited aperture case. Left column: geometry settings. The source point is denoted by the small red circle, while the receivers are denoted by the small blue stars. The sound-soft, sound-hard obstacles and the inhomogeneous medium are coloured in black, blue and yellow, respectively. The black dashed line depicts the boundary of the sampling region. Middle column: images of $I(z ; y) / \max _{z}\{I(z ; y)\}$. Right column: images of $I_{\mathrm{TFM}}(z ; y) / \max _{z} I_{\mathrm{TFM}}(z ; y)$. We take $y=(-3,0)$. 


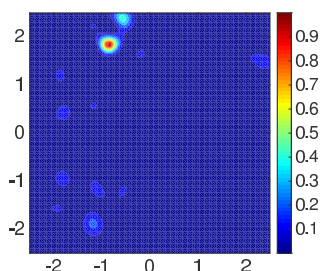

(a)

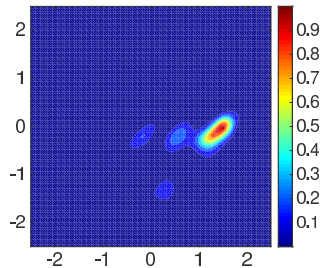

(d)

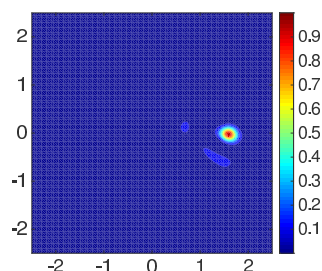

(b)

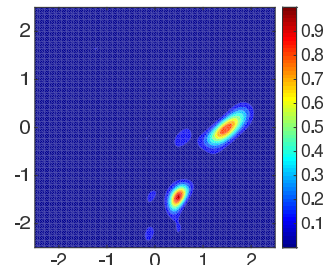

(e)

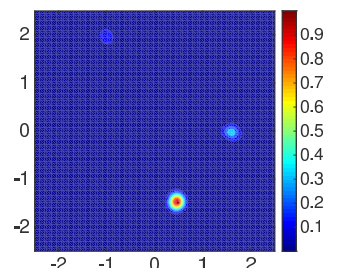

(c)

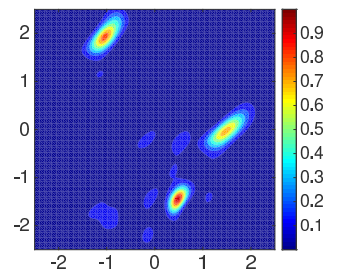

(f)

Figure 3: Reconstruction of small scatterers using the dynamic indicator $\tilde{I}(z, t) / \max _{z} \tilde{I}(z, t)$. Top row: full aperture case of Figure 2 (a). Bottom row: limited aperture case of Figure $2(\mathrm{~d})$. These snapshots are captured at: (a) $t=4.22$. (b) $t=6.86$. (c) $t=8.06$. (d) $t=6.86$. (e) $t=7.76$. (f) $t=9.02$.

scatterer component, which is in accordance with our theoretical analysis. Using partially measured data, Figure 2 (e) (f) show that the location of the small scatterers can be less precisely captured than using the full data; cf. Figure 2 (b) (c). In this case it is typical that the point spread functions are somehow elongated, due to the lack of information in inversions. The bottom-left scatterer component can be more clearly imaged in Figure 2 (e) (f), because its location is closer to the receivers than others.

Figure 3 illustrates the evolutionary behaviour of the dynamic indicator function (12) by showing the snapshots of the reconstructions at some special stages. As can be seen from Figure 3, the dynamic indicator exhibits a selective focusing property such that the global maximum point of the indicator flows from one scatterer position to another. An advantage of this capability is that, in our view, the retrieval of relatively weak scatterer components (for instance the sound-hard scatterer in this example) may be readily achieved compared to the stationary plots. For a comparison, we refer to Figure 3 (b) vs. Figure 2 (b) (c), and Figure 3 (d) (e) (f) vs. Figure 2 (e). Note that the bottom-left scattering component does not appear in Figure 3 (a)-(f), because the terminal time $t$ in our dynamic indicator is not taken sufficiently large (see Proposition 1 for the indicator behavior).

\subsection{Reconstruction of crack-like scatterers with at most two sources}

In this section, the exact scatterer is chosen as a crack-like object which has a rectangular shape with height 0.1 and width 2 such that it is "partially small". 
The impedance boundary condition with $\lambda=0.1$ is imposed on the boundary of the scatterer. Synthetic scattered data were collected with terminal recording time $T=12$ and 600 recording time steps. We aim to test the sensitivity of the reconstructions to the orientation of this slender rectangle using a single source and the effect of normalization involved in (15) for two incident sources.

In the extreme case that the orientation of the crack coincides with the radical direction of the point source (see Figure 4 (a)), only the locations of the endpoints can be retrieved using the single source scheme (11), whereas the center part is invisible; see Figure 4 (b) (c). With such images one would probably argue that the exact scatterer consists of two disconnected point-like objects only rather than a crack. From the physical perspective, this "misleading" result is due to the weak scattering from the center part of the crack. If we fix the crack and change the point source location, or alternatively, fix the source location but adjust the crack orientation, then the center part of the crack could be recovered; see Figure 4 (e) (f) and Figure 4 (h) (i). However, the true scatterer seems to be two adjacent and parallel straight cracks rather than a single one, as can be seen from Figure 4 (e) and Figure 4 (h). From the physical point of view, a possible explanation to this effect is that the close twins in the reconstruction are originated from the close opposite side of the crack. Evidently, the proposed single-source indicator could be used for recovering a crack-like scatterer and gives better images than the TFM.

We remark that the single-source indicators have been scaled to $[0,1]$ in Figure 4 (b) (h). It is shown in Figure 5 (b) (d) that the exact magnitudes of the indicators due to different source locations may vary significantly. Figure 6 highlights the effect of normalization in the case of two sources where singlesource indicators are summarized. Without normalization, the contribution of a certain source to the superposition might be overwhelmed by other sources (see Figure $6(\mathrm{~b})$ ).

\subsection{Reconstruction of regular-size scatterers with multi- sources}

In this example, the underlying scatterer consists of a disc component $(c=5)$ and an L-shaped component $(c=3)$. Because of the insufficient information obtained, measurements due to a few point source may expose the incapability of imaging the full geometrical features of an extended scatterer. Hence, we employ multiple excitation points to illuminate the unknown scatterer from different directions in order to capture more geometrical details of the target object.

In Figure 7 we compare the reconstructions using the three multi-source indicators proposed in Section 4.3. For regular-size scatterers, it seems that the indicator $I(z)$ and the total focusing method $\tilde{I}_{\mathrm{TFM}}(z)$ provide images with almost the same resolution. However, the indicator $\tilde{I}_{\text {NEW }}(z, t)$ for some sufficiently large $t$ could yield better results than $I(z)$ and $\tilde{I}_{\mathrm{TFM}}(z)$, especially in recovering the concave part of the L-shaped component which cannot be illuminated by some point source waves and where multiple scattering occurs. However, all indicators show the incapability of precisely imaging the concave part. Figure 8 illustrates the snapshots of the dynamic indicator $\widetilde{I}_{\mathrm{NEW}}(z, t)$. It is concluded from Figure 8 (a)-(d) that the boundaries of the underlying scatterer that are far-away from point sources can be earlier retrieved than other 


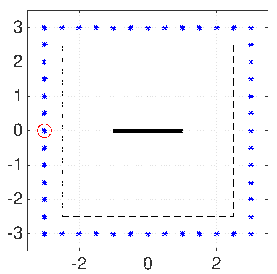

(a)

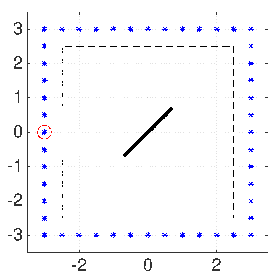

(d)

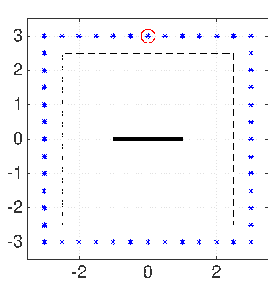

$(\mathrm{g})$

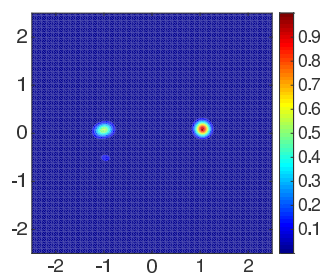

(b)

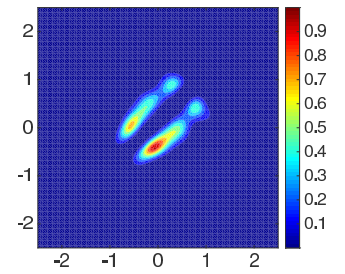

(e)

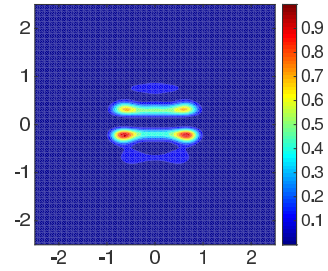

(h)

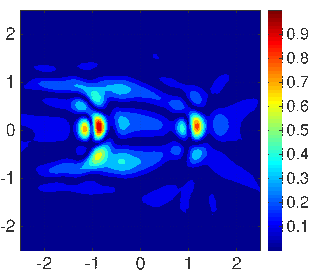

(c)

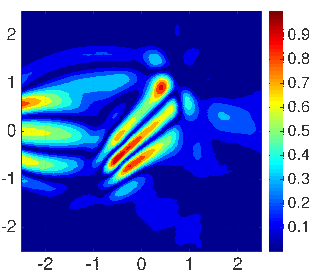

(f)

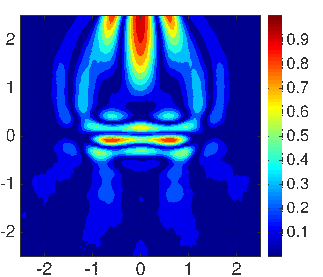

(i)

Figure 4: Reconstruction of cracks. Row 1-3: examples of the cracks with different orientations, respectively. The source point is denoted by the small red circle and the receivers are denoted by the small blue stars. Left column: exact cracks. Middle column: images of $I(z ; y) / \max _{z}\{I(z ; y)\}$. Right column: images of $I_{\mathrm{TFM}}(z ; y) / \max _{z}\left\{I_{\mathrm{TFM}}(z ; y)\right\}$. 


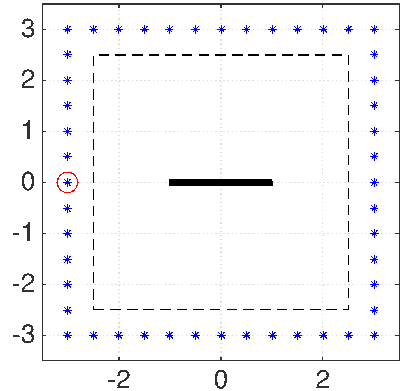

(a)

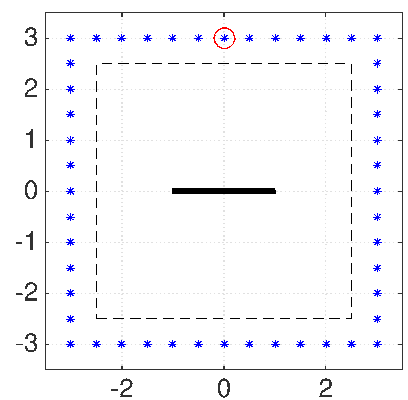

(c)

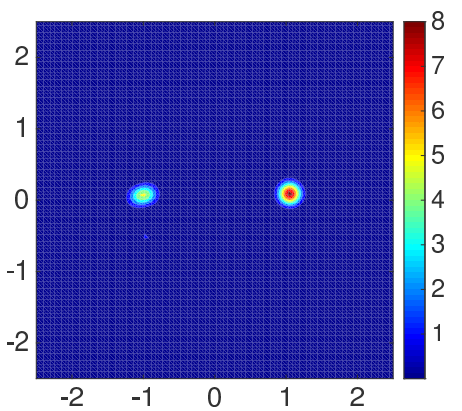

(b)

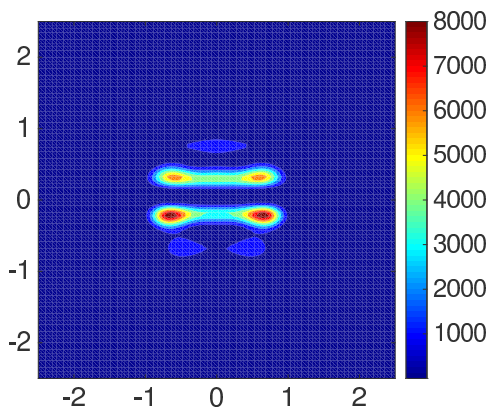

(d)

Figure 5: Reconstruction of a crack using different sources. The geometry settings are shown in (a) and (c), respectively. The reconstructions are, respectively, the images of $I(z ; y)$ with $y=(-3,0)$ in $(\mathrm{b})$ and $y=(0,3)$ in $(\mathrm{d})$.

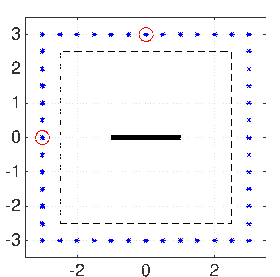

(a)

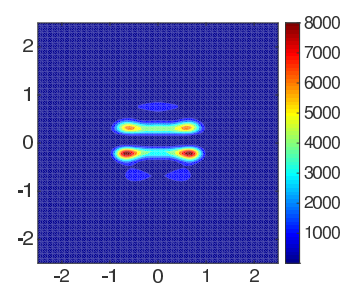

(b)

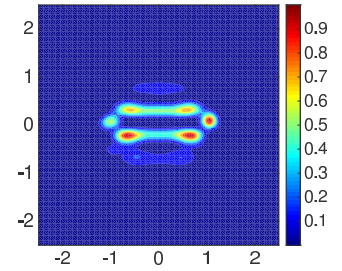

(c)

Figure 6: Reconstruction of cracks using two sources. (b) the image of $I\left(z ; y_{1}\right)+$ $I\left(z ; y_{2}\right)$ with $y_{1}=(-3,0), y_{2}=(0,3)$. (c) the image of the normalized indicator $I\left(z ; y_{1}\right) / \max _{z} I\left(z ; y_{1}\right)+I\left(z ; y_{2}\right) / \max _{z} I\left(z ; y_{2}\right)$. The geometry setting is shown in (a). 


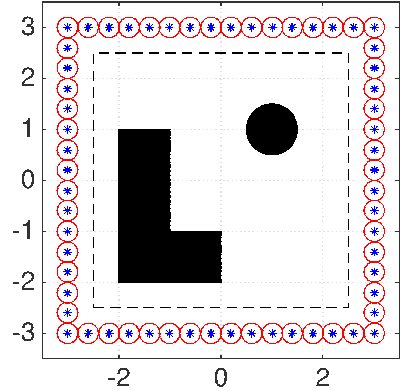

(a)

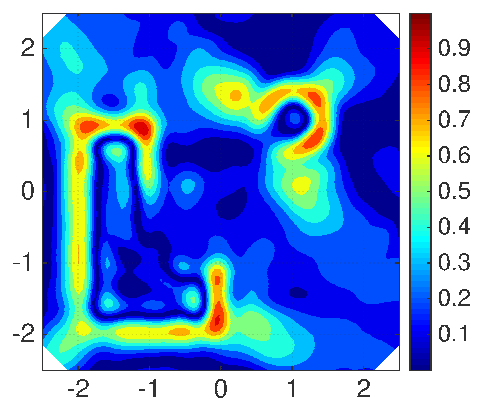

(c)

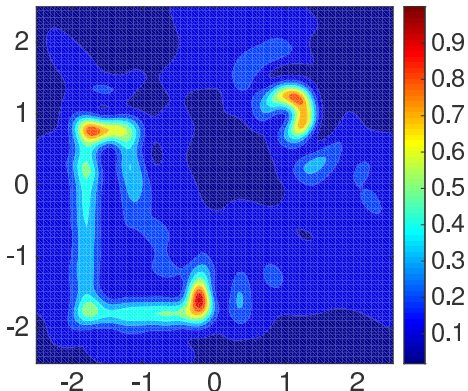

(b)

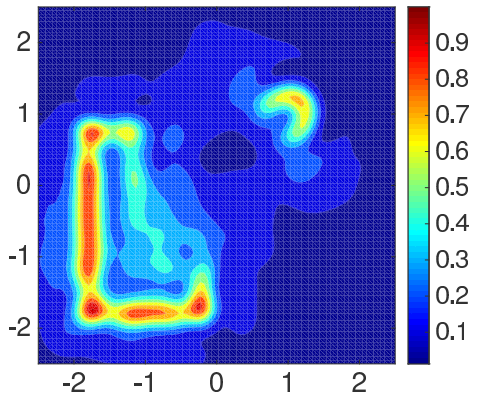

(d)

Figure 7: Reconstruction of regular scatterers using different indicator functions with 64 sources. (a) geometry settings. The source points are denoted by the small red circles and the receivers are denoted by the small blue stars. (b) image of $I(z) / \max _{z} I(z)$. (c) image of $I_{\mathrm{TFM}}(z) / \max _{z} I_{\mathrm{TFM}}(z)$. (d) image of $\tilde{I}_{\mathrm{NEW}}(z, t) / \max _{z} \tilde{I}_{\mathrm{NEW}}(z, t)$ with $t=5.86$.

parts, which is in agreement with the theoretical analysis performed in Theorem 1 for point-like scatterers.

\subsection{Reconstruction of regular-size scatterers in three di- mensions}

The last example is devoted to the reconstruction of a three-dimensional regularsize scatterer. To our knowledge, this is the first numerical realization of the proposed schemes and the TFM for imaging three-dimensional regular-size scatterers.

In this example, the exact scatterer consists of two penetrable disconnected cubes with sound speed $c=2$ inside. We use 56 sources to emit the incident pulse and 56 receivers to collect the scattered data. The recording time array has 300 time steps with terminal time $t=19$. The true scatterer and the sources/receivers are illustrated in Figure 9 (a) (b).

To view the reconstruction, we present both the slice plot and the iso-surface plot of the volumetric indicator functionals. In the slice plots, the images are 


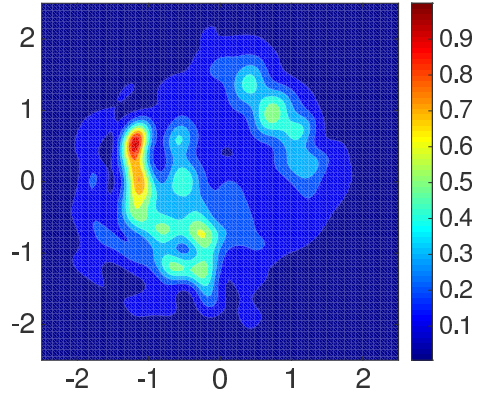

(a)

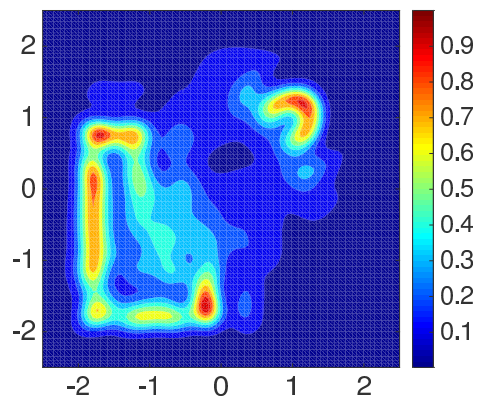

(c)

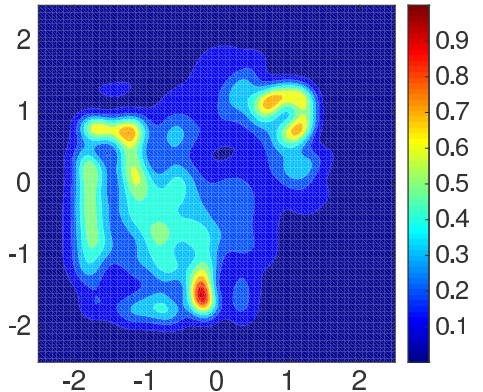

(b)

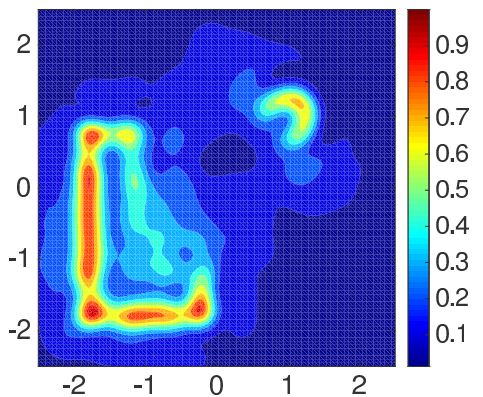

(d)

Figure 8: Reconstruction of regular scatterer using the dynamic indicator $\tilde{I}_{\mathrm{NEW}}(z, t) / \max _{z} \tilde{I}_{\mathrm{NEW}}(z, t)$. These snapshots are captured at: (a) $t=3.78$, (b) $t=4.74$, (c) $t=5.06$ and (d) $t=5.86$. 
drawn with two slices through the center of the cubes along the $x z$ - and $x y$ plane. In the iso-surface plots, the images are drawn according to suitable iso-surface levels which are chosen by trial and error. See Figure 9 (c)-(f) for the reconstructions.

\section{Conclusion}

We propose several non-iterative reconstruction methods to solve inverse scattering problems for time-dependent acoustic waves. Some preliminary mathematical analysis for point-like scatterers is carried out and the effectiveness of the static and dynamic indicators is illustrated via a variety of numerical examples in two and three dimensions. The proposed imaging schemes can be regarded as the strengthened total focusing method. For the first time we examine the performance of such direct sampling schemes for recovering crack-like and regular-size scatterers. Our future work consists of the mathematical justification of the indicator behaviors for imaging regular-size scatterer and the extension of the approach to the more practical case of elastodynamic wave equations.

\section{Acknowledgement}

Y. Guo would like to thank the hospitalities of the South University of Science and Technology of China and Weierstrass Institute for visiting positions. The work of Y. Guo was supported by a grant from the National Natural Science Foundation of China (NNSFC) under No. 41474102 and No. 91230119. The work of H. Liu was supported by the FRG grants from Hong Kong Baptist University, Hong Kong RGC General Research Funds, 12302415 and 405513, and the NSF grant of China, No. 11371115. The work of J. Li was partially supported by the NSF of China (No. 11201453, 11571161), Guangdong research fund (No. K11243101), and Shenzhen Sci-Tech fund (No. JCYJ20140509143748226).

\section{References}

[1] H. Ammari, J. Garnier, and K. Sølna, A statistical approach to target detection and localization in the presence of noise, Waves in Random and Complex Media 22 (2012), 40-65.

[2] H. Ammari, E. Iakovleva, D. Lesselier and G. Perrusson, MUSIC-type electromagnetic imaging of a collection of small three-dimensional inclusions, SIAM J. Sci. Comput., 29 (2007), no. 2, 674-709.

[3] H. Ammari and H. Kang, Reconstruction of Small Inhomogeneities from Boundary Measurements, Lecture Notes in Mathematics, Vol. 1846, Springer-Verlag, Berlin 2004.

[4] E. Baysal, D. D. Kosloff and J. W. C. Sherwood, Reverse time migration, Geophysics, 48 (1983), 1514-1524.

[5] M. I. Belishev, Boundary control in reconstruction of manifolds and metrics (the BC method), Inverse Problems, 13 (1997), R1-R45. 


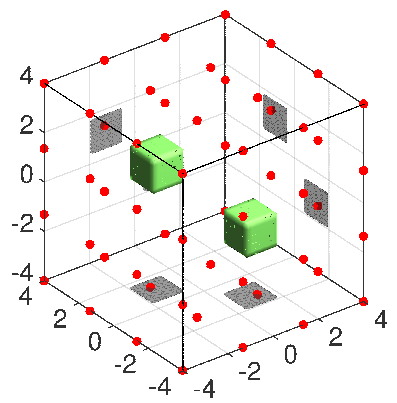

(a)

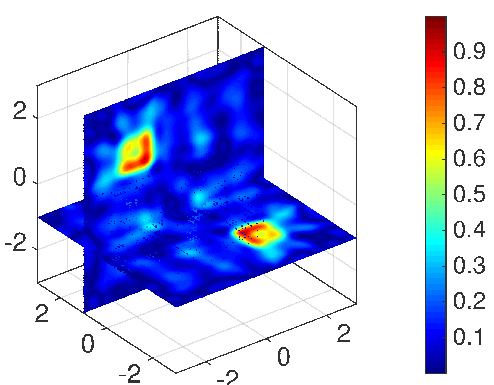

(c)

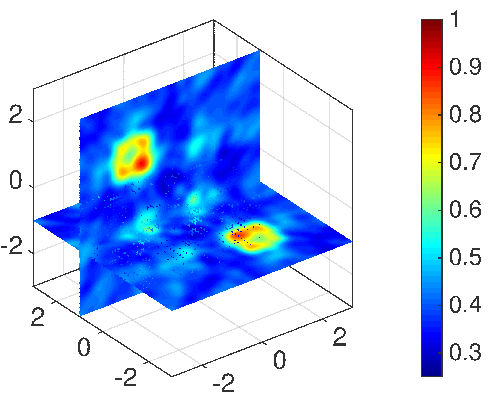

(e)

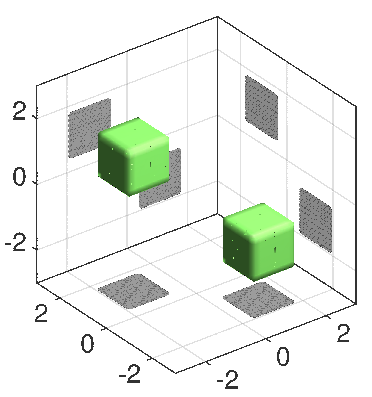

(b)

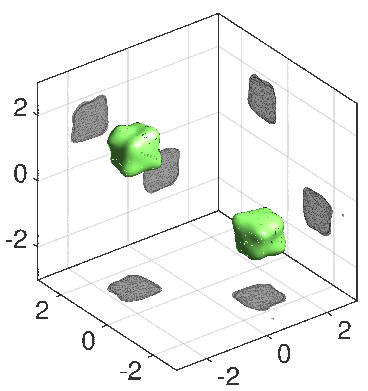

(d)

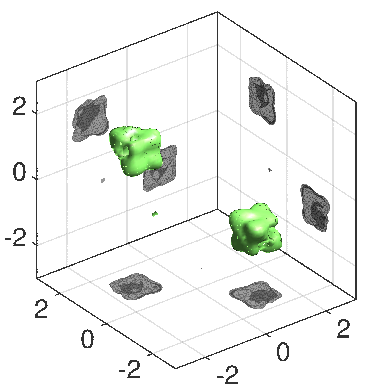

(f)

Figure 9: Reconstruction of two disconnected cubes. (a) geometry setting. The small red balls denote the locations of sources and receivers. (b) exact scatterer. (e) slice plot of $I_{\mathrm{TFM}}(z) / \max _{z}\left\{I_{\mathrm{TFM}}(z)\right\}$. (d) iso-surface plot of $I_{\mathrm{TFM}}(z) / \max _{z}\left\{I_{\mathrm{TFM}}(z)\right\}$ with iso-surface level 0.45. (e) slice plot of $I(z) / \max _{z}\{I(z)\}$. (f) iso-surface plot of $I(z) / \max _{z}\{I(z)\}$ with iso-surface level 0.7 . 
[6] L. Beilina and M.V. Klibanov, A globally convergent numerical method for a coefficient inverse problem, SIAM J. Sci. Comp., 31 (2008), 478-509.

[7] L. Beilina and M.V. Klibanov, Approximate global convergence and adaptivity for coefficient inverse problems, Springer, New York, 2012.

[8] C. Burkard and R. Potthast, A time-domain probe method for threedimensional rough surface reconstructions, Inverse Problems and Imaging, 3 (2009), 259-274

[9] D. Colton and A. Kirsch, A simple method for solving inverse scattering problems in the resonance region, Inverse Problems, 12 (1996), 383-393.

[10] D. Colton and R. Kress, Inverse Acoustic and Electromagnetic Scattering Theory, 3rd Edition, Springer-Verlag, Berlin, 2013.

[11] A. J. Devaney, Super-resolution processing of multi-static data using time reversal and MUSIC, J. Acoustic Soc. Am., 2000.

[12] Y. Guo, D. Colton and P. Monk, Toward a time domain approach to the linear sampling method, Inverse Problems, 29 (2013), 095016.

[13] M. Fink, Time reversed acoustics, Physics Today 50 (1997), 34.

[14] J.-P. Fouque, J. Garnier, G. Papanicolaou, and K. Sølna, Wave Propagation and Time Reversal in Randomly Layered Media, Springer, New York, 2007.

[15] C. Holmes, B. W. Drinkwater and P. D. Wilcox, Post-processing of the full matrix of ultrasonic transmit-receive array data for non-destructive evaluation, NDT\& E International, 38 (2005), 701-711.

[16] M. Ikehata, Inverse scattering problems and the enclosure method, Inverse Problems, 20 (2004), 533-551.

[17] M. Ikehata, Extracting the geometry of an obstacle and a zeroth-order coefficient of a boundary condition via the enclosure method using a single reflected wave over a finite time interval, Inverse Problems, 30 (2014), 045011.

[18] V. Isakov, Inverse obstacle problems, Inverse Problems, 25 (2009), 123002.

[19] M.V. Klibanov and N.T. Thánh, Recovering of dielectric constants of explosives via a globally strictly convex cost functional, SIAM J. Appl. Math., 75 (2015), 518-537.

[20] A. Kirsch and N. Grinberg, The Factorization Method for Inverse Problems, Oxford Lecture Series in Mathematics and Its Applications, Oxford University Press, Oxford, 2008.

[21] Y. Labyed and L. Huang, Ultrasound time-reversal music imaging with diffraction and attenuation compensation, IEEE Trans. Ultrason., Ferroelectrics and Frequency Control, 59 (2012), 2186-2200.

[22] O. A. Ladyzhenskaya, Boundary Value Problems of Mathematical Physics, Springer, New York, 1985. 
[23] J. Li, H. Liu, Y. Sun and Q. Wang, Ground detection by a single electromagnetic far-field measurement, J. Comput. Phys., 273 (2014), 472-487.

[24] J. Li, H. Liu and J. Zou, Locating multiple multiscale acoustic scatterers, SIAM J. Multiscale Model. Simul., 12 (2014), 927-952.

[25] D. R. Luke and R. Potthast, The point source method for inverse scattering in the time domain, Math. Meth. Appl. Sci., 29 (2006), 1501-1521.

[26] L. Oksanen, Solving an inverse obstacle problem for the wave equation by using the boundary control method, Inverse Problems, 29 (2013), 3500435015 .

[27] R. Potthast, A fast new method to solve inverse scattering problems, Inverse Problems, 12 (1996), 731-742.

[28] R. Potthast, A study on orthogonality sampling, Inverse Problems, 26 (2010), 074015.

[29] N. T. Thành, L. Beilina, M. V. Klibanov and M. A. Fiddy, Imaging of buried objects from experimental backscattering time dependent measurements using a globally convergent inverse algorithm, SIAM J. Imaging Sciences, 8 (2015), 757-786.

[30] C. H. Wilcox, Scattering Theorey for the d'Alembert Equation in Exterior Domains, Springer-Verlag, New York, 1975. 